\title{
LUZERNE ET APICULTURE
}

PAR

\section{J. LECOMTE}

Station de recherches apicoles. Bures-sur-Yvette

I,es relations entre la I, uzerne et 1'Abeille sont très complexes. Ifon peut les considérer, soit du point de vue de l'apiculteur qui est évidemment intéressé par les possibilités de récolte, soit du point de vue du producteur de semences qui recherche une augmentation de rendement par l'intensification de la pollinisation.

Ce dernier point de vue se révèle d'ailleurs en dernière analyse, le plus important, puisque, outre les avantages que la pollinisation procure aux producteurs de semences, elle amène fatalement ceux-ci à partager leurs bénéfices avec les apiculteurs. Une juste rémunération du travail effectué par l'Abeille remplace ainsi la récolte de miel que la Ituzene n'est pas toujours en mesure de fournir.

La première question que nous poserons sera justement:

\section{I a-1-il du miel de luzerne?}

Dans un travail récent, Maurizıo (1958) décrit quelques miels contenant du pollen de I,uzerne et rencontrés par elle au cours de ses analyses polliniques. Elle n'a jamais rencontré de miels contenant plus de 52 p. roo de pollen de luzerne et le miel sur lequel a été faite cette observation était un miel d'origine inconnue. Un certain nombre de miels originaires de Californie présentent un pourcentage de pollen de Luzerne non négligeable : 7 possèdent entre $r_{3}$ et 34 p. I0o de ce pollen. Toujours d'après Maurizio, l'on peut citer un miel canadien qui atteint I 5 p. Ioo et un miel français qui en contient I4 p. Ioo.

De toute manière, ces miels se sont révélés très paurres en pollen et il y a lieu d'appliquer un coefficient de correction, non encore connu, mais très certainement considérable. MAURIzIo (I958) est ainsi d'avis que l'on peut déclarer un miel de Iłuzerne " pur " même quand le pourcentage de pollen de cette espèce n'atteint que 45 p. Ioo. 
Iin Irance, d'indiscutables miels de Luzcrne ont été récoltés dans les régions de polder de la baie dı Mont Saint-Michel (I,ouveatr, renseignement oral), dans les polders de l'Onest-Atlantique (Rimbardo, I057) et anssi dans d'autres régions, bien que les suppositions des apiculteurs n'aient pas été toujours contirmées par l'analyse pollinique.

Ín ce qui concerne l'abondance de la miellée, I)ADAN' (I95I) signale avoir observé aux U. S. A. des récoltes moyennes de $9 \mathrm{~kg}$ par ruche; celles-ci étant au nombre de douze par hectare. STEPHEN (I955) signale unc observation de P.ANkIW qui, toujours anx Ftats- $T$ nis, pour une concentration de ruches analogue, aurait observé des récoltes de 0 ¿i ro $\mathrm{kg}$. Ces récoltes, notons-le, semblent ninines aux auteurs anéricailis.

Ein U. R. S. S. Sovor,iva (I952), a observé mue récolte moyenne de $5 \diamond 3 \mathrm{~g}$. Il semble donc qu'il puisse y avoir récolte de nectar sur les fleurs de I,uzerne ; cependant la miellée n'a lieu que dans certaines conditions encore mal connues et d'autre part, la plupart des autres plantes mcliifères détournent très facilement les butineuses des champs de I, 11zerne.

F'RANkLIN (I95I) signale qu'au Kansas les principanx compétiteurs sont le Mélilot et P'olygomium persicaria. PAxkIw et collaborateurs (I956), etrent beancoup de difficultés à faire butiner de la I,uzerne à cause de la présence de Montardes. I ans cet exemple, if p. Ioo seulement des butineuses se dirigcaient vers l's champs de I, nzerne. STrpHex (i 9.55) signale que dans les régions arides des litats-Unis, il est très facile de faire activement butincr les onvière; sur les I,uzernes parce qu'il n'existe pas d'autres fleurs ; il en est do mêne clans les terres à blé canadiennes où les luzernes sont isolées an milieu de grandes étendues de céréales sans intérêt pour l'Abeille.

\section{Les abeilles et lo pollen de luzerne.}

'Lots les auteurs sont d'accord pour reconnaitre l'absence ou la très grande rareté des pelotes de pollen de I,tuerne. Citons par exemple I,OUVEAUX (I958) ; STIPIIEN (I955), BLAGOVLIIMNKAYA (1957), ÁkERBERG et LESINS (I049), Mc MAHON (I054), qui enl Irance, aux U. S. A., en U. R. S. S., enl suède, et au Canada ont fait les mênes observations. L,e ramassage le plus important a été observé en T R. S. S. par BogoravIXNSKII (I95.3) qui a constaté que 7,4 p. roo des butineuses d'un champ) portaient atx pattes des pelotes, d'ailleurs rudinentaires.

Avant de nous interroger sur les causes de cette inappétence, il convient d'envisager comment s'effectue le butinage de la fleur de I,uzerne. 


\section{Le butinage de la fleur de luzerne.}

I a fleur de I,11zerne comporte cinq pétales, n11 que l'on nomne l'étendard, une paire qui forme les deux ailes et wne autre paire qui constitue la carène. Cette carène renferme les étamines et le pisti1. Pour qu'il puisse Y avoir fécondation, la carène doit s'écarter et laisser les organes sexuels se libérer. Quand un insecte écarte les deux constituants de la carène, les organes sexuels se relèvent brusquement et viennent frapper l'étendard avec force. Ce déclenchement est la phénomène connu des anteurs anglosaxons sous le nom de "tripping"; c'est en I 867 qu'il fut, semble-t-il pour la première fois, cécrit par HeNsion (fig. I).

La plupart des auteurs estiment que le "tripping" est indispensable pour que la fleur soit fécondée; I 1). Ioo seulement des fleurs n'ayant pas subi le déclenchement peuvent fructifier (Kxowiss, I043).
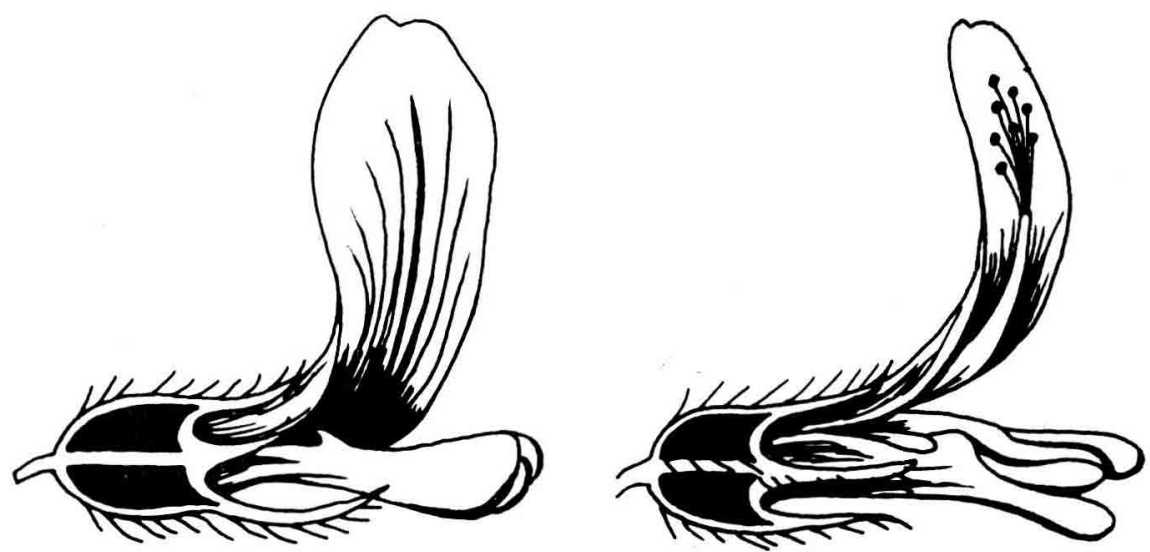

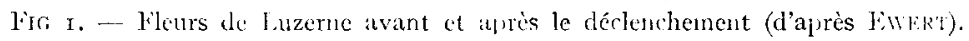

Au moment oì la colonne sexuelle est libérée par une Abeille butineuse, un petit paquet de pollen est déposé à la base du proboscis de celle-ci.

D’après GRINFIL'D (I956), 78 p. Ioo des abeilles présentes sur un champ de luzerne portent ainsi du pollen à la base du proboscis, ce qui démontre qu'elles ont au moins une fois provouué le déclenchement d'une fleur. İEIN (I955) de son coté, a observé un pourcentage plus élevé : go p. IOo ; ce signe permet d'ailleurs de reconnaître les butineuses de I,uzerne lors de comptages effectués au trou de vol. I)'ailleurs, selon VANSELL (I955), les butineuses de nectar portent ce signe caractéristique tout autant que les butineuses de pollen, ce qui peut expliquer les pourcentages élevés signalés par les auteurs.

I,e choc ressenti par l'abeille lors du déclenchement est universellement considéré comme la cause principale du peu d'empressement mis par l'Abeille à récolter le pollen de la I, uzerne. 
Dès I873, MULIER avait vu que la butineuse de nectar cherchait à éviter le déclenchement. 'Tandis que les autres hyménoptères, lorsqu'ils provoquent le déclenchement, dirigent l'impact sur le côté de la tête, neutralisant ainsi le choc, selon GRINFFI'D (I956), 1'Abeille, au contraire après avoir subi ce petit traumatisme, modifie son travail. REINHART (I952) a étudié ce comportement particulier; les butineuses de nectar évitent le déclenchement en insérant leur trompe entre l'étendard et les ailes, et de ce fait leur vitesse de travail augmente considérablement. Les butineuses de pollen, pour autant qu'il en existe, apprennent à effectuer le déclenchement sans être atteintes par la colonne sexuelle.

\begin{tabular}{|c|c|c|}
\hline Insectes & $\begin{array}{c}\text { 'Taux } \\
\text { de declenchement } \\
\text { en p. Ioo } \\
\text { de fleurs visitées }\end{array}$ & Auteur \\
\hline 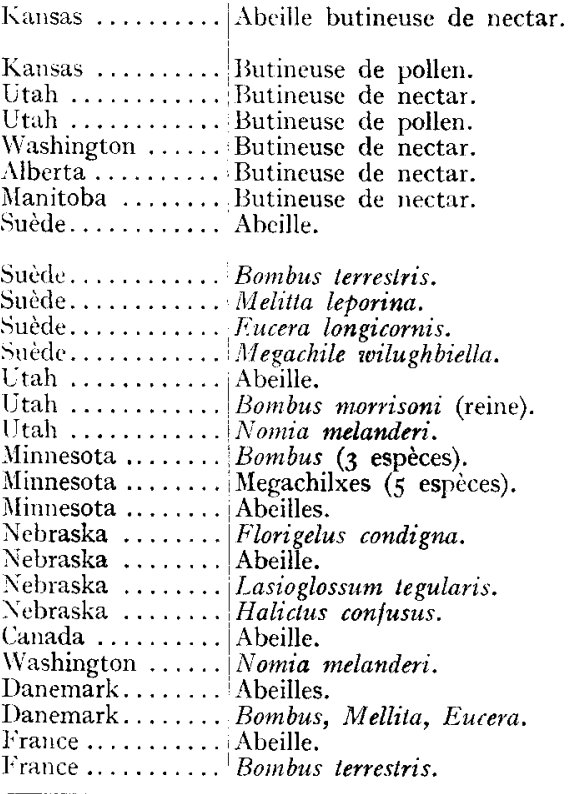 & $\begin{array}{c}65 \\
17,5 \\
59 \\
0,4 \\
0,81 \\
0,10 \\
0,83 \\
78 \\
97 \\
100 \\
100 \\
0,14 \\
24 \\
11,8 \\
34 \\
97,5 \\
0,4 \\
.30 \\
2 \\
0 \\
0 \\
0,25-1,8 \\
95 \\
0,4-1,6 \\
89-98 \\
1,5 \\
99\end{array}$ & 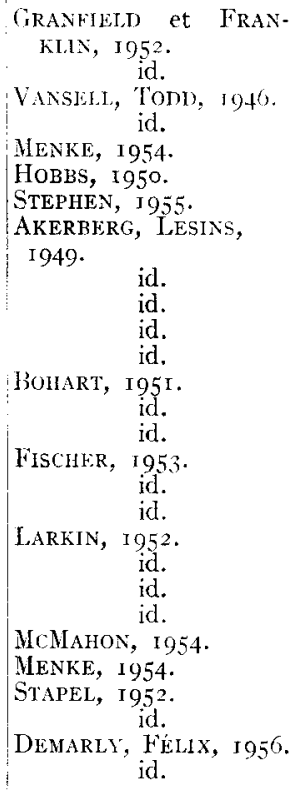 \\
\hline
\end{tabular}

Avec Vansel, et ToDn (I946), nous distinguerons donc: les butineuses de pollen, les butineuses de nectar par voie frontale et les butineuses de nectar par voie latérale. Ces dernières sont de beaucoup les plus nombreuses et leur rôle serait nul en ce qui concerne la pollinisation s'il n'était prouvé qu'elles effectuent parfois le déclenchement accidentellement, avec leurs pattes. (HoBr et LILLY, I955, STEPHEN, I955).

Néanmoins, le pourcentage global des visites pendant lesquelles les Abeilles effectuent le déclenchement est très faible.

Le tableau précédent condense quelques résultats se référant à des 
taux de déclenchenent observés par différents auteurs, non seulement sur l'Abeille, mais aussi, à titre de comparaison sur différents hyménoptères visiteurs de la I,uzerne.

En ce qui concerne ces derniers, un tableau donné par BOH.ART (I956) fournit des renseignements analogues pour 70 espèces d'hyménoptères du monde entier.

\section{L'abeille est-elle d'un intérêt quelconque en tant qu'agent pollinisateur?}

Un très faible tanx de déclenchement a été si souvent constaté chez l'Abeille que nombre d'auteurs mettent en doute sa valeur en tant qu'agent pollinisateur. Cependant, un grand nombre de faits prouvent qu'il n'en est rien et que des gains appréciables peuvent être réalisés dans les récoltes de graines par l'emploi des Abeilles. Aux I'. S. A. la rareté ou la diminution du nombre des Hyménoptères sauvages met en évidence de manière spectaculaire la valeur de 1'Abeille. Selon (GROTT (I950), la récolte moyenne était avant I930 de I 400 ooo $\mathrm{kg}$ dans l'Ttah. A cette date, la transhumance des Abeilles est interdite pour des raisons sanitaires; la récolte tombe à $340000 \mathrm{~kg}$; en I932, elle n'est plus que de 75 ooo $\mathrm{kg}$. L'embargo est levé en $\mathrm{I} 934$ et cette année la récolte atteint I $600000 \mathrm{~kg}$.

Des essais systématiques corroborent par ailleurs cette impression d'ensemble.

Tout d'abord, hâtons-nous de discréditer complitement les différents essais effectués en cage et en serre. Il est absolument certain que le taux de déclenchement dans ces conditions est tout à fait anormal. ÅKERBERG et JESINs (r949) ont montré que dans ces conditions artificielles, le taux de déclenchement des butineuses devenait aussi élevé que celui des meilleures espèces de Bombus. Penterseñ et Boharí (I950) ont constaté le même phénomène che $z$ des Bourdons. Des espèces qui normalement ne récoltent que du nectar sur la I,uzerne, ramassent également du pollen dans ces conditions artificielles.

Seuls les essais effectués en plein champ devront être retenus. Citons 111 travail de DADAN' (I95I). I2 colonies à l'hectare furent employées et ces colonies furent changées tous les dix jours pour éviter l'effet répulsif du déclenchement auquel les Abeilles, nous le savons, apprennent très vite à réagir, par une visite latérale de la fleur. 1)ans ces conditions, alors que la récolte moyenne de 1'État de Californie n'était que de $300 \mathrm{~kg}$ à l'hectare, elle fut dans les champs d'expérience de I $232 \mathrm{~kg}$. Pankiw, Boltow, Mc MAHON et FoST'ER (I956) ont comparé des parcelles d'une acre de superficie à proximité desquelles se trouvaient $0,1,2$ et 3 ruches. Bien que II p. Ioo seulement de la population des butineuses se soient dirigées 
vers les parcelles de Luzerıe (à cause de la présence de fleurs concurrentes) les récoltes moyennes par acre furent de 35, 45, 72, in livres anglaises. Menke (I952-54), cependant met ell évidence que dans l'État de Wrashington, l'action des abeilles est très reduite par rapport à celle des " Abeilles Alkali ": Nomia melanderi.

Penersen (I953) a mis en évidence une corrélation positive asse\% élevée entre le nombre de visites d'abeilles et le nombre de graines. VANSELI. (I95I), a également montré que la quantité de graines était directement liée au nombre de ruches présentes. Haws (1950), étudiant des parcelles d'une acre de superficie sur lesquelles se trouvaient 4 ruches a trouvé d'évidentes corrélations entre le nombre de graines d'une part et d'autre part, la qualité du terrain, le nombre d'abeilles, leur activité et l'absence de fleurs compétitives.

Enfin, GRANFIELD (I954), a calculé à partir d'observations précises que 5 à 7 ruches à 1'hectare visitent de 356 à 1067 millions de fleurs en un jour et qu'étant donné la longue période de floraison de la I,uzerne ( au Kansas elle est de 20 jours en moyenne) l'on peut obtenir une pollinisation tris satisfaisante, même avec un pourcentage de déclenchement très bas Finalement, malgré leur très faible pourcentage de déclenchement, ce sont les butineuses de nectar, les plus nombreuses de loin, qui sont responsables de 1'accroissement des récoltes (VANSF, I, I95 I).

Citons enfin 1'opinion de JAmieson (r950) pour qui les Abeilles sauvages sont plus importantes que les Abeilles domestiques pour la pollinisation de la I,uzerne au Canada, à moins que ces dernières ne soient en grand nombre et qu'il n'y ait pas de compétitions avec d'autres plantes. I)e toute manière, le travail de JonEs (I950) pronve que la présence d'un grand nombre d'abeilles sur un chanp de Ituzerne n'anènt. pas une diminution du nombre des autres hyménoptères et 1'Abeille ne saurait ainsi a voir qu'un rôle favorable ou neutre. Cet avis n'a d'ailleurs pas toujours été partagé, d'autres auteurs, PINGEILY (1954), P'Ack et BOLTON (I946) pensent que dans certains cas, la présence d'abeilles écarte les autres insectes.

Une très abondante littérature existe d'ailleurs sur les différents hyménoptères pollinisateurs de la I,uzerne; nous renvoyons le lectenr désireux de s'informer au travail de BOHART' (I957) déjà cité et pour la Russie à Porov (I952 et I956).

\section{Peut-on améliorer le travail des abeilles sur la luzerne?}

Il faut d'abord faire remarquer que les différents clones de Luzerne semblent présenter des différences significatives quand à leur attractivité Pedersen et Todo (I949) en étudiant, en serre, I8 clones, ont trouvé des taux de déclenchenent variant de 30 à 86 p. Ioo lors de visites effectuées 
par des Abeilles. Notons que ces taux, comme nous l'avons déjà fait remarquer, sont très exagérés par rapport à ceux que l'on peut espérer rencontrer dans la nature. Cependant, les différences subsistent et ont sans doute une signification.

PeDersen et Bohart (I950) ont également trouvé des différences semblables en effectuant les tests avec Bombus morrisoni. Pour ces auteurs des corrélations existent entre l'attractivité d'une part et la concentration en sucre du nectar et la teneur en carotène des feuilles d'autre part. Selon IEVIN et PEDERSEN (I955), les Abeilles seraient bien plus sensibles encore que les Bourdons à ces facteurs d'attractivité.

D'après Louveaux (I958), il faut attribuer le peu d'appétit des Abeilles pour le pollen de I uzerne, non seulement à l'effet du "tripping" mais aussi à une carence en principes attractifs généralement présents dans les autres pollens. Il faut en effet remarquer que le pollen de I uzerne est riche en azote et pourrait de ce fait, intéresser les butineuses. LEvIN et BOHART (I955) avaient déjà postulé qu'un facteur inconnu, composition chimique ou odeur, était responsable des différences d'attractivité entre les différents pollens.

Pour Bogoyavinenski (I953), le nombre de visites est également lié à l'abondance de la sécrétion et à sa teneur en sucre. Des données précises existent sur le rôle de la teneur en sucre pour différents nectars (voir par exemple VANSELI, I952), mais il semble que dans le cas de la Iuzerne, d'autres facteurs rentrent en jeu. FRANKI,IN (I95I) a observé que le Mélilot détourne les Abeilles de la I,uzerne bien que la concentration en sucre du nectar de cette dernière fût de 0,5 à 5 p. Ioo supérieure à celle du nectar du Mélilot.

Il est donc important de supprimer les plantes compétitrices chaque fois que la chose est possible. Cette suppression doit cependant être aussi complète que possible. LINSLEY et Mac SwaIN (I947) ne sont arrivés à détourner des Abeilles d'un champ de Moutarde vers la I,uzerne qu'après que le champ de moutarde entier eût été coupé.

Par ailleurs, il existe non seulement des différences d'attractivité entre les différents clones de Luzerne mais encore des différences entre les manières de se comporter des différentes races d'Abeilles. Les taux de déclenchement peuvent ainsi varier considérablement, (ÅkERBERG et I ESINS I949). PETERSEN (I954) trouve au Danemark une supériorité de l'Abeille noire sur l'italienne et dans l'Oklahoma, selon BIEHERdori: (I949), la caucasienne serait supérieure à l'italienne.

Ces données encore fragmentaires indiquent cependant qu'il serait dangereux de considérer la Luzerne ou l'Abeille comme une entité indivisible et les différences existant entre les différentes variétés ou races demandent certainement à être précisées.

Une autre question importante est celle de l'osmo-guidage des A beilles 
sur lu Luzerne. Von Frisch (I947) dans son ouvrage de base "Duftgelenkte Bienen im Dienste der Iandwirtschaft und Imkerei " ne cite que quatre essais concernant cette plante. De ces quatre essais, deux sont positifs et deux négatifs. En effet, les ruches ayant reçu un sirop de nourrissement parfumé à la fleur de Iruzerue ont montré des différences de récolte par rapport aux ruches témoins, respectivement de + I5 p. roo, + I60 p. I00; - 28 p. I00; - 9 p. Ioo. Dans les quatre cas, il s'agit de la moyenne de trois ruches. Blagoveshchenkaya (r95.5) en plaçant des Abeilles près d'un champ de Luzerne et en effectuant un osmoguiđage n'obtient pas une amélioration du taux de déclenchement. Même si l'on retire tous les cadres de pollen de la ruche, les Abeilles cherchent à reconstituer leurs provisions de préférence aux dépens des plantes compétitives.

Sovolfiva (I952) a nourri des ruches avec une infusion de fleurs de Luzerne dans du sirop de sucre. Le maximun de visites eut lieu de 3 à 5 jours après le nourrissement. Durant cette période, le nombre d'Abeilles butineuses sur le champ expérimental était de I I à I4 fois plus élevé que sur le champ témoin. Dans ces conditions, Sovor Eva a constaté que le plus grand nombre des butineuses se trouvaient dans un rayon de 500 mètres autour du rucher, aucune n'était observable au-delà de I ooo mètres.

Ce fait permit d'apprécier le gain en semence dû̀ à la présence des butineuses; tandis que la récolte à $500 \mathrm{~m}$ des ruches est de $310 \mathrm{~kg}$, elle n'est à I 200 mètres que de $8 \mathrm{I} \mathrm{kg}$.

Par ailleurs, les Abeilles ainsi guidées, récoltent en moyenne I o4I grammes de nectar par jour, tandis que les témoins n'en récoltent que 583.

Tzygankov (I953) a effectué des observations similaires en Asie Centrale. Avant l'osmoguidage, 90 p. roo des Abeilles travaillaient à moins de 400 mètres de la ruche et $9 \mathrm{p}$. Ioo à plus de 400 et moins de I 000 . Après l'osmoguidage, le pourcentage devint voisin de $37 \mathrm{p}$. Ioo à Iooo mètres. Dans un essai où furent employés Ix 6 colonies pour 30 hectares de luzerne, le rendement par hectare, à proximité immédiate du rucher, fut de $247 \mathrm{~kg}$, tandis qu'entre I et I,5 km du rucher, il n'était plus que de $127 \mathrm{~kg}$. En associant le retrait des cadres de pollen de la ruche à un osmoguidage pratiqué avec du sirop de sucre parfumé au pollen de I,uzerne, RUDNEV (I94I) a considérablement augmenté l'activité de récolte du pollen, mais cette augmentation a porté sur tous les pollens plus que sur le pollen de I uzerne seul.

Il a pu sembler important d'opérer avec des colonies n'ayant pas l'expérience de la luzerne. Nous avons déjà signalé que les abeilles apprenaient à éviter le déclenchement. LEVIN (I955) a observé cependant que des abeilles d'une colonie placée, depuis peu, près d'un champ le visitaient plus volontiers qu'une colonie depuis un certain temps en place; mais ceci 
est vrai que les nouveaux venus provienment ou non d'une région où se trouvait de la luzerne. Pour I IEvin et BOHAR'l (I957), le transport des ruches permet d'obtenir de bons résultats seulement s'il se fait d'une région où domine la luzerne vers une région où celle-ci ne constitue qu'une partie de la flore apicole.

Il ne faut pas oublier non plus que les éléments les plus divers peuvent rentrer en ligne de compte. PEDERSEN et Mc ALIISTER (I953) ont pu ainsi constater que les luzernières clairsemées ont deux fois plus de nectar, deux fois plus de visites d'Abeilles et deux fois plus de graines par fleur que les luzernières très denses.

\section{Conclusions.}

Malgré tune somme de travaux considérable, le problème est loin d'être résolu.

Nul doute que 1'Abeille puisse jouer un rôle dans la pollinisation de la Ituzerne, mais que ce rôle soit toujours suffisamment important pour justifier l'apport de ruches à proximité des luzernes, cela n'est pas certain.

Un certain nombre de conditions sont sans doute requises pour que 1'Abeille travaille au mieux et dans certains cas il est sans doute préférable de compter sur les hyménoptères sauvages et de rechercher les moyens propres à en conserver et à en augmenter les populations.

Provisoirement et en attendant que les recherches effectuées en France aient apporté les adaptations nécessaires aux conditions locales, l'on peut adopter les recommandations formulées par ToDD et VANSELL (I952), chaque fois que les conditions externes laissent pressentir une possibilité d'utilisation de l'Abeille.

Ces recommandations peuvent se résumer ainsi :

Si les butineuses de pollen semblent abondantes, il suffit d'environ 6 à 7 ruches par hectare; si elles sont rares, ce qui est le cas le plus fréquent une quinzaine de colonies sont indispensables pour une même superficie.

Il est recommandé de disposer les ruches par paquets de ro à $\mathbf{r} 2$ à l'intérieur des champs. Enfin, les butineuses n'ayant pas encore l'expérience de la Luzerne, ayant un taux de "déclenchement " plus élevé, l'on se trouvera bien d'amener les colonies en plusieurs fois durant la période de floraison.

\section{RÉFÉRENCES BIBLIOGRAPHIQUES}

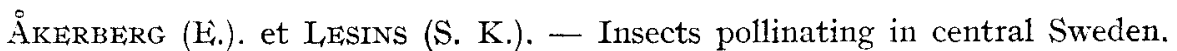
Kungl. Lantbrutshösk. Ann., 16, 630-643, I949.

Blagoveshchenskaya (N. N.). - Des abeilles forcées à polliniser la Iruzerne (en russe). Pchelovodstvo, 9, 5I-53, r955. 
Bogoyavienskit (S. G.). -- Abeilles et Inzerne (en russe). Pchelovodstro, 6, 36-4I, I953.

BorrarT (G. E.). - Alfafa seed growers of Utah should protect their wild bees. F arm and home Sci., 12, (2), 32-37, I95I.

Bohart (G. E.). - Pollinisation of Alfafa and red Clover. Ann. Rev. Entom., $2,355-380,1957$.

Dadant (M. G.). - Alfafa seed-one thousand pounds per acre. American Bee $J ., 91,(4)$, I $42-I 43$, I95I.

DEMARLY (Y) et FÉLIX (L.). - Observations sur la production des graines de I,uzerne. Bulletin technique d'information des ingénieurs des services agricoles., 106, 3-15, I956.

FIscher (R. L.). - Native pollinators of Alfafa in northern Minnesota. Minn. Beekpr., 6, (3), 8-9, r953.

Franki, IN (W. W.) - Insect affecting alfafa seed production in Kansas. Techn. Bull. Kans. Agric. Exp. Sta., 70, 64 p., I95I.

FRISCH (K. von). - Duftgelenkte Bienen im Dienste der Landwirtschaft und Imkerei. Springer Verlag. Vienne, I89 p., I947.

Grandfield (C. O.). - Alfafa in Kansas. Techn. Bull. Kans. Agric. Exp. Sta, 346, 65 p., I95I.

Granlited (C. O.), Franklin (W. W.). - Alfafa seed production in Kansas. Circ. Kans. Agric. Exp. St., 290, 28 p., I952.

GRINFEL'D (E. K.). - Évaluation du rôle de l'abeille dans la pollinisation de la Luzerne. (en russe) Pchelovodstvo, 33, (6), 32-35, I956.

Grout (R. A.). - Pollination. An agricultural practive. Hamilton III, Dadant \& Sons 23 p., r950.

Haws (B. A.). - Honeybee activity and other environmental factors as related to alfafa seed production. Iowa Report State Apiarist for, I950, $57-58$

Hensiow (G.). - Proc. Linnean Soc. Botany, 9, 327-29, I866.

Hosbs (G. A.). - Pollinating species of bees in the irrigated regions of southern Alberta. Report. Twelfth Alfafa improvement cont., Lathbridge. Alberta., 47-49, I950.

Hobrs (G. A.) et Lricy (C. E.). - Can, J. Agr. Sci., 35, 422-432, I955.

JAMreson (C. A.). - Investigation at the bee division. Canad. Bee Journal., $58,(2), 20-22,1950$.

Jones (L. M.). - Rept. I2 th Alfafa Improvement Conf., 38-40, I950.

Knowles (R. P.). - Sci. Agr., 24, 29-50, I943.

LARKIN (R. A.). - Wildbee populations cycle and the production of Alfafa seed. Agron. J., 44, (4), 2I6-2I8, I952.

LEVIN (M. D.). - A technique for estimating the percentage of honey bees visiting Alfafa. J. Ent., 48, (3), 484-85, I955.

IEVIN (M. D.) et BohaRT (G. E.). - The effect of prior location on alfafa foraging by honey bees. J. econ. Entomol. U. S. A., 50, (\$), 629-632, I957.

I.EVin (M. D.) et PEDERSEN (M. W.). - A technique for collecting pure Alfafa pollen. Agron. J., 4\%, (8), 387-88, I955.

Lrinsi,ey (E. G.) et MacSwain (J. W.). - J. Econ. Entomol. U. S. A., 40, 349-57, I947.

Louveaux (J.). - - Recherches sur la récolte du pollen par les abeilles (Apis mellifica L.) Thèse Paris, I958, 206 p.

McMahon (H.). - Pollination of Alfafa by honeybees. Canad. Bee J., 62 (3), 4-6, I954.

Maurizio (A.). - Beitraege zur quantitativen pollenanalyse des Honigs. Annales de l'A beille, 1, (2), 93-106, I958.

Menke (H. F.). - Alkali bee helps set seed records. Crops and soils, 4, (8), I952.

Menke (H. F.). - Insect pollination in relation to Alfafa seed production in Washington. Wash. Agr. Expt. St. Bull. No $555, \mathrm{r} 954$. 
MÜLLER (H.). - Die Befruchtung der Blumen durch Insekten und die gegenseitigen Anpassungen beider. Leipzig, I873.

Pankiw (P.), Borton (J. L.), McMahon (H. A.) et Foster (J. R.). - Alfafa. pollination by honey bees on the regina plain of Saskatchewan. Canad. J. agric. Sci., 36, (2), I I4-II 9, I 956.

Peck (O.) et BolTON (J. L.). - Sci. Agr., 26, 388-4I7, I946.

PEDERSON (M. W.). - Seed production in alfafa as related to nectar production and Honeybee visitation. Bot. Gaz., 115, (2), I29-I38, I953.

PEDERSFN (W. M.) et BohaR'T (G. E.). - Factors responsible for the attractiveness of various clones of Alfafa to pollen-collecting Bumble bees. A gron. J., 45, 548-5 I, I953.

Pedersex (M. W.) et Me Airister. - Utah Agr. Expt. St. Circ., I35, 60 p., I 955 .

Penersen (W. M.) et ToDd (F. K.). - Selection and tripping in Alfafa clones by nectar collecting honeybees. Agron. $J ., 41,(6), 247-49$, I949.

Pengeity (D. H.). - 48 th ann. Rept. Entomol. Soc. Ontario., IOI-II8, I954.

Petersen (H. I.). - Arsskrift. Kgl. Vet. og Landbohôiskole. I38-I69, I954.

Porov (V. B.). - Le role des Abeilles (Hymenoptera-A poideae) dans la pollinisation de la Luzerne. (en russe). All-Union Entomol. Soc, 43, 65-82, r952.

Popov (V. B.). - Les Abeilles, leurs relations avec les plantes mélittophiles et le problème de la pollinisation de la Luzerne. (en russe). Entomol. Revs, U.S.S.R., 35, 528-98, I956.

REINHARDT (J. F.). -- Some responses of honey bees to Alfafa flowers. Amer Nat., 86, (830), 257-275, I952.

Rimbaud (E.). - La vie d'un rucher pendant 34 ans. Revue Fr. d'A piculture, 53-56, février 1957 .

RUDNEV (W.). - Socializtichezkove Zemovoye Khozhaiztvo, 2, I4I-I44, I94I.

Sovoreva (E. M.). - Les Abeilles et la production de graines de Luzerne. (en russe) Pchelovodstvo, 8, 39-4I, I952.

STEPHEN (W. P.). - Alfafa pollination in Manitoba. J. econ Entomol. U. S. A., 48, $543-548$, I955.

Todd (F. E.) et VAnsei (G. H.). - Proc. 6 th Intern. Grasslands Congr., 1, 835-840, I952.

'Tzyankov (S. K.). - Pollinisation par les abeilles de la Luzerne destinée aux semences. (en russe) Pchelovodstvo, 6, 33-36, I953.

VANSELL (G. H.). - Use of Honeybees in Alfafa seed production. Circ. U.S. Dpt. A gric., No 876, II pages, I95I.

VANSELL (G. H.). - Variations in nectar and pollen sources affect bee activity. Amer. Bee J., 92, 8, 325-326, 1952.

VAnsei, , (G. H.). - Alfafa pollen on nectar-collecting Honey bees. J. econ. Entomol. U.S. A., 48, (8), 477, I955.

VAnsell (G. H.) et Todd (F. E.). - Alfafa tripping by insects. J. Americ. Soc. Agrom., 38, 470-487, I946. 